\title{
Recurrent and density-dependent patterns in long-term fluctuations of Atlantic bluefin tuna trap catches
}

\author{
F. Royer ${ }^{1,2, *}$, J. M. Fromentin ${ }^{1}$ \\ ${ }^{1}$ IFREMER, Centre de Recherche Halieutique Méditerranéen et Tropical, avenue Jean Monnet, BP 171, 34203 Sète cedex, France \\ ${ }^{2}$ Present address: Large Pelagics Research Lab, University of New Hampshire, Durham, New Hampshire 03824, USA
}

\begin{abstract}
We present a methodology for the non-linear analysis of long-term ecological time series. Using a time-delay embedding procedure, we investigated the complexity of 1-dimensional signals and compared their structure to their stochastic counterparts. Recurrence-based statistics and surrogate testing were used for this purpose. The method was first tested on elementary models for illustrative purposes and its potentialities and limits reviewed. We then investigated the time structure of 6 Mediterranean bluefin tuna trap catches known to display long-term fluctuations. These time series showed significantly more determinism than linear stochastic processes, displaying more recurring patterns/states than stochastic surrogates. The short term structure of these series also showed a direct and delayed negative feedback in their rate of increase. These results were homogeneous over all studied time series. An interaction between climate forcing and the life history traits of bluefin tuna was postulated to explain the first result, while the density-dependent pattern may be explained either by density-dependence processes during early stages, or by non-successive (nonyearly) spawning events in the Mediterranean.
\end{abstract}

KEY WORDS: Thunnus thynnus $\cdot$ Recurrence $\cdot$ Density-dependence $\cdot$ Surrogate testing $\cdot$ Embedding

\section{INTRODUCTION}

Studying variability in ecological systems over time and space is an extensive area of research which has gained considerable interest in recent years (Gurney \& Nisbet 1998, Turchin 2003). Quantifying the relative importance of biotic and abiotic processes on free-ranging marine populations has, however, been hampered by several limitations, including: (1) the lack of long ecological time series, (2) the presence of observation errors, especially when populations are sampled blindly, and (3) the complexity of the interactions between deterministic and stochastic mechanisms (Ripa et al. 1998, Cazelles \& Boudjema 2001, Fromentin et al. 2001, Turchin 2003). When conducting exploratory data analysis, the alternation and combination of stochastic and deterministic periods, which possibly involve non-linear interactions and non-stationary noise, renders many standard linear methods inadequate (such as the Fourier- and correlation-based approaches, Dale et al. 2002). Moreover, fitting standard population models often supposes prior knowledge of the underlying mechanisms and that any 'uninformative' noise is simply additive (uncorrelated) and can be assimilated to observation error. Recent studies have, however, demonstrated that these assumptions are not often valid: large-scale coherent patterns can emerge from stochastic internal processes (e.g. from interactions between individuals or age classes, Bjornstad et al. 2004), while environmental noise tends to be autocorrelated over space and time (especially in marine ecosystems, Steele 1985, Vasseur \& Yodzis 2004). Lastly, little information may be available on the processes that govern population dynamics over the studied time scales. 
In this context, Atlantic bluefin tuna Thunnus thynnus L. poses a challenging problem. The exploitation of this pelagic migratory species dates back to antiquity, and has reached critically high levels during the past decades (see Fromentin \& Powers 2005 for a review). Despite continuous documentation efforts, detailed information on key processes (i.e. migratory behaviour, population structure, habitat use, growth and reproduction) required to elaborate and fit an accurate population model is still not available. Furthermore, extreme events, such as bursts and/or disappearance of bluefin tuna in given areas, have been reported in the past (Tiews 1978, Mather et al. 1995), while apparent shifts in spatial distribution and/or migration continue to be an actual and acute problem (Lutcavage et al. 1999, Fromentin \& Powers 2005). These observations suggest that the dynamics of bluefin tuna may include several potential mechanisms operating at different spatial and temporal scales. The recent collection of long time series from the coastal trap fisheries in the Mediterranean greatly helped in describing patterns of variations, which were largely dominated by low-frequency signals (Ravier \& Fromentin 2001). In this previous study, we showed that medium- to long-term fluctuations ( 20 and $\sim 100$ to $120 \mathrm{yr}^{-1}$ ) were particularly synchronous over the whole studied area (>2000 km), whereas inter-annual variability was only synchronous at a local scale. Using linear regression techniques and stochastic simulation, Fromentin (2002) and Ravier \& Fromentin (2004) proposed a set of hypotheses to explain these mediumto long-term fluctuations (from time-delayed effects related to a long life span and numerous age classes to climate-induced effects), but we did not investigate the potential causes of inter-annual variability.

In this study, we performed a reanalysis of a set of these historical time series to: (1) extract patterns of variability that may have remained hidden when using linear techniques, and (2) examine whether we could further discriminate between deterministic and stochastic processes. For this purpose, we applied a nonlinear exploratory technique, namely recurrence analysis (RA) (Zbilut et al. 2002). RA is based on recent strategies derived from the signal processing and chaos theory field and was first applied to various issues in optics, finance, physiology, hydrology and chemistry (Eckmann et al. 1987, Marwan et al. 2002). It relies on quantifying self-similarity in the data and detecting recurring patterns over time, with no assumptions on their statistical nature. RA can thus characterize the dynamical properties of the observed system, regardless of its non-linearity or non-stationarity (Gao \& Cai 2000). Such a technique appears particularly suited for complex ecological systems, for which little mechanistic knowledge exists and where only 1 vari- able is measured (e.g. abundance or catch, as is the case here). There has been few applications of this technique in ecology: Dippner et al. (2002) employed RA to uncover deterministic (periodic) fluctuations and non-linear interactions in a controlled mesocosm experiment, while Parrott (2004) showed how RA could help in visually detecting disturbances in a simulated ecosystem.

As RA is still relatively new to ecology, we here describe the basic steps of this technique (i.e. phasespace embedding, recurrence plotting and recurrence quantification) and then apply it to simple examples for illustration. We then show how the use of surrogate data may help to extract ecological information from RA outputs. A specific limitation of RA was identified in the case of finite, noisy data (a common situation in ecology) and an additional step based on partial correlation (Berryman \& Turchin 2001) was proposed to address this issue. Finally, we applied RA to time series of Atlantic bluefin tuna trap catches and present results on previously hidden patterns. These new findings are discussed in the light of current knowledge on Atlantic bluefin tuna's intriguing population dynamics, and new modes of variability are proposed.

\section{MATERIALS AND METHODS}

Recurrence analysis. Phase-space embedding: Ecologists often study population dynamics using a small number of observed variables, often no more than 1 (e.g. abundance, density or catch). Such data can be greatly informative under a basic assumption, i.e. that the sampled period is sufficiently long to encompass most of the system's possible states. It is then possible to reconstruct the dynamics of hidden processes from a single time series, thus uncovering some aspects of their structure; this can be done using time-delay embedding or equivalently time-delay coordinates. Let us write the population size at time $t, N_{t}$, as a function $f$ of its past abundances (of time order $d$ ):

$$
N_{t+1}=f\left(N_{t}, N_{t-1}, N_{t-2}, \ldots, N_{t-d}\right)
$$

Such an approach is common in quantitative ecology and can be seen as a generalized delay-difference population model where neither the exact relationship $f$ nor the order $d$ are known. If $f$ is considered linear, an autoregressive model (of an estimated order $d$ ) could be fitted to the data (Box \& Jenkins 1976). In the non-linear case, embedding and recurrence analysis provide a tool to characterise the complexity and shape of $f$ (Boudjema \& Chau 1996, Zbilut et al. 2002).

We must first define what a 'proper' embedding is. Given the time series of measures $N_{t}$, with $t=1,2,3, \ldots$, $T$, an embedding procedure will form a new series of 
points $X_{i}$ of coordinates $\left(N_{i,} N_{i+L}, \ldots, N_{T-(m-1) L}\right)$ with $m$ the embedding dimension and $L$ the lag. The succession of $X_{i}$ now represents the multidimensional process of the time series as a trajectory in a $m$-dimensional space, subsampled every $L$ time step. The lag, $L$, or time delay, corresponds to the memory of the process. $L$ must be chosen so as to avoid neighbouring points being too close in the $m$-space and to maximize the information provided by each measure. The embedding dimension $m$ relates to the complexity (the order) of the hidden ecological process. For example, a simple 2-dimensional embedding will detect patterns in the growth rate of a single population (Turchin 2003). Higher dimensions will reveal more complex dynamics and interactions with other variables or pseudo-cyclic features (more points being needed to 'capture' such oscillations). Very high dimensions (15 to 20+) are indicative of high order randomness, i.e. we would be unable to capture their dynamics through a finite number of equations (see Hsieh et al. 2005). Takens' theorem (Takens 1981) states that optimal $m$ and $L$ values will yield a one-to-one mapping between the reconstructed phase-space and the phase-space of the hidden system. In other words, the dynamics of the reconstructed system are the same as the dynamics of the original true system: characteristic invariants are preserved (e.g. the number of controlling variables), thus allowing identification of the possible processes underlying a noisy, apparently random data series. In practice, $L$ is chosen as the first minimum in the average 'mutual information' function (which quantifies the dependence between states taken at different lags), while $m$ is derived using the Euclidean false nearest neighbours method (see Kantz \& Schreiber 1997).

The recurrence plot: A qualitative description of the observed dynamics can be obtained by plotting the (m-dimensional) states that repeat themselves in the data and which are called recurrent states (Eckmann et al. 1987). A recurrence plot (RP, Zbilut et al. 2002) is constructed by placing a point at coordinates $(i, j)$ on matrix of size $[T-(m-1) L, T-(m-1) L]$ whenever a state $X_{i}$ on the trajectory is considered close to another state $X_{j}$. The closeness between $X_{\mathrm{i}}$ and $X_{j}$ is expressed as the Euclidean distance $\left\|X_{i}-X_{j}\right\|<r$, where $r$ is a fixed radius. The resulting matrix is symmetrical (a point at $[i, j]$ is also present at $[j, i]$ ), with the $x$ - and $y$-axes being the new time coordinates. RPs display patterns that can be qualitatively interpreted (Gao \& Cai 2000, Parrott 2004). For example, diagonals are formed by different parts of the trajectory that evolve in a similar manner: such portions repeating themselves after a certain period of time indicate deterministic behaviour. Horizontal and vertical lines indicate states that do not change over successive time steps. Isolated dots, on the other hand, are likely to indicate states that repeat themselves by chance (randomly). However, RPs remain hard to read, and a formal description and quantification of these patterns is needed for further analysis. The value of $r$ may also affect their sensibility to observation noise, and the overall number of detected recurrences; this could be a limitation, and we addressed it later in this section.

Recurrence quantification: The structure of an RP is usually defined through a number of statistics (Gao \& Cai 2000, Zbilut et al. 2002): (1) the recurrence rate (\%REC) is the ratio of all recurrent states to all possible states, and is therefore the probability of the recurrence of a certain state $X_{i i}(2)$ the determinism (\%DET) is the ratio of recurrence points forming diagonals to all recurrence points, and shows the likelihood of having a piece of trajectory close to another at different times; (3) the average diagonal length (ADL) is the average time span that diagonals will be close to each other and thus can be interpreted as a mean prediction time; (4) the average and longest diagonal length (ADL and LDL): the inverse of the longest diagonal length is related to the largest Lyapunov exponent of the system (a measure of its predictability, Eckmann et al. 1987); (5) laminarity (LAM) quantifies the amount of vertical and horizontal lines in the RP, thus characterising the probability of having the system 'locked' in a certain state; and finally (6) the trapping time (TT) measures the average time spent in such laminar phases (Marwan et al. 2002). These statistics (or probes, in the sense of Turchin 2003) are of great value for characterizing the complexity of non-linear dynamics, as their interpretation is relatively straightforward. Table 1 summarizes these 7 statistics and their interpretation.

Hypothesis testing using surrogates: The statistics describing the recurring points and patterns on an RP can then be used for hypothesis testing against elementary random processes. The method of surrogates

Table 1. Names, acronyms and definitions of statistics used in recurrence analysis (RA) (from Gao \& Cai 2000, Zbilut et al. 2002)

\begin{tabular}{|c|c|c|}
\hline Name & Acronym & Interpretation \\
\hline $\begin{array}{l}\text { Recurrence } \\
\text { rate }\end{array}$ & $\%$ REC & $\begin{array}{l}\text { Ratio of all recurrent states } \\
\text { to all possible states }\end{array}$ \\
\hline Determinism & $\% \mathrm{DET}$ & $\begin{array}{l}\text { Ratio of diagonal recurrent } \\
\text { states to all recurrent states }\end{array}$ \\
\hline $\begin{array}{l}\text { Average } \\
\text { diagonal length }\end{array}$ & $\mathrm{ADL}$ & $\begin{array}{l}\text { Mean prediction time of } \\
\text { time series }\end{array}$ \\
\hline $\begin{array}{l}\text { Longest } \\
\text { diagonal length }\end{array}$ & LDL & $\begin{array}{l}\text { Largest prediction time of } \\
\text { time series }\end{array}$ \\
\hline Laminarity & LAM & $\begin{array}{l}\text { Quantity of laminar phases (same } \\
\text { state occurring consecutively) }\end{array}$ \\
\hline Trapping time & TT & $\begin{array}{l}\text { Average time spent in } \\
\text { laminar phases }\end{array}$ \\
\hline
\end{tabular}


is used here for the detection of determinism or highly recurring patterns. Linear surrogate techniques have been introduced by Theiler et al. (1992) to determine whether experimental time series are consistent with various classes of simple linear systems. Each surrogate is consistent with a specific null hypothesis about the underlying system (e.g. a random variable or an autoregressive-like process) while retaining at the same time some of the statistical features of the original time series (e.g. mean and variance, power spectrum, complete empirical distribution). Statistics are computed for the original data and the surrogates, thus allowing to test (in a statistically rigorous way) if the data is consistent with the null hypothesis.

This method thus relies on both the algorithm employed to generate the surrogates and the ecological relevance of the hypothesis one wants to test for characterising the system (Royama 1992, Hilborn \& Mangel 1997, Gurney \& Nisbet 1998). The surrogates discussed in the following section are based on various 'colored' noise models (by analogy with the light spectrum, see e.g. Halley 1996). 'White noise' refers to a time series spectrum where all frequencies are equally represented, meaning that successive values are uncorrelated. A 'blue noise' implies that the time series spectrum is dominated by short-term fluctuations (high frequencies), whereas 'reddened noise' are dominated by long-term fluctuations (low frequencies). Some phenomena are known to have deeply reddened spectra, leading to 'brown' or even 'black' noises (Cuddington \& Yodzis 1999). Note that most environmental noises are reddened, especially in marine systems (Steele 1985, Vasseur \& Yodzis 2004).

The simplest null model would be that the data originates from 'white noise' (i.e. an independent and identically distributed variable, possibly non-gaussian). Corresponding surrogates are often generated by simply shuffling the data, thus preserving the original empirical distribution but destroying any time correlation. This algorithm is often referred to as 'Type 0 ' in the literature (Theiler et al. 1992, Small \& Tse 2003) and is mainly seen as an autocorrelation test of the data prior to more advanced testing. It is worth noting that the white noise null hypothesis is generally unsuited to geophysical or ecological problems, because few processes are able to generate white noise outputs (Hasselmann 1976, Rohani et al. 2004). In ecological time series, white noise is often restricted to the observation process. However, assuming a white noise for the dynamical process itself (e.g. population growth) is equivalent to specifying the null model of an almost perfectly compensated population (as the mean and variance remain constant, see Royama 1992, Berryman \& Turchin 2001).

A more complex null model ('Type 1 surrogate') corresponds to the generation of 'reddened noise', which is the usual way of trying to capture endogenous fluctuations in populations (Royama 1992). In such case, the linear properties of the time series are fully described by its autocorrelation function or equivalently by its power spectrum. Surrogate data are therefore build by adding random phases in $[0,2 \pi]$ to the components of the Fourier transform of the observed time series, and then computing its inverse Fourier transform (Theiler et al. 1992). The resulting surrogates are Gaussian and have the same mean, variance and power spectrum as the original data. Applying this algorithm to a time series of abundance is particularly interesting when attempting to detect regulation (i.e. density-dependence) in population dynamics, as it is equivalent to the null model of an unregulated population growth (Berryman \& Turchin 2001). Testing against coloured noise further allows the assessment of the significance of pseudo-oscillations/trends in the data, since univariate autoregressive processes cannot per se support deterministic low-frequency oscillations (Rohani et al. 2004). Statistical testing of series with reddened spectrum is still the subject of research and debate in ecology, as many tests rely on the assumption of white noise and lose their power for autocorrelated or non-stationary series (see Morales 1999, Rudnick \& Davis 2003).

An even more complex null model ('Type 2 surrogate') will preserve both the original non-Gaussian distribution and the power spectrum of the data (V. Venema et al. unpubl. data). Such surrogates can be generated using the amplitude adjusted fourier transform (AAFT) algorithm. Such calculation slightly distorts the spectrum, but generally yields to satisfying surrogates for sufficiently long data sets, e.g. more than 100 points (Schreiber \& Schmitz 1996). This is equivalent to producing non-Gaussian data from an autoregressive process combined with a monotonic and static non-linear observation function. Strictly speaking, any non-linearity here is non-dynamic, i.e. attributed to the measurement function. Such formulation is very convenient in time-series analysis, and ecological studies in general, where a major task is to separate sampling error from dynamical noise (Turchin 2003).

For each test, the generation of a large number of surrogates allowed the derivation of a distribution of the RP-based statistics. As most distributions were non-Gaussian, the significance level was assessed in a non-parametric manner, i.e. by counting up the fraction of realizations that produce a value greater than the observed one. The threshold $\geq\left(2 \alpha^{-1}-1\right)$ was chosen, where $\alpha$ is the residual probability of false rejection (see Marwan 2003). All the calculations were performed using Matlab 6.5 (Matlab 2003), and the calculation of the RPs statistics were achieved using code made available by N. Marwan et al. (www.agnld. 
uni-potsdam.de/ marwan/toolbox/) and V. Venema (www.meteo.uni-bonn.de/mitarbeiter/venema/themes/ surrogates/).

Observation noise and the choice of $r$-limitations and solutions: We noted that time-delay embedding allows the retrieval of real dynamics of a partially observed system in the noise-free case or for true (additive) observational noise only, but that there is no guarantee that noise-driven dynamics will be completely reconstructed. Previous studies have, however, shown that this technique can be powerful for diagnostic and identification purposes, when there is only a little knowledge on the underlying processes of a system (Boudjema \& Cazelles 2001).

In the application case of finite, noisy time series (a common feature in marine ecology where observational errors are common), the choice of $r$ may limit the detection of high frequency patterns; for example, 2 recurring system states $X_{i}$ and $X_{j}$ could be infinitely close, but measurement error combined with a small $r$ value would misclassify them as non-recurring. On the other hand, a more permissive (larger) $r$ value would increase the number of apparent recurring states in the system, and thus the risk of false detections. We addressed this by: (1) using the same (arbitrary small) $r$ values for all series and corresponding surrogates, and (2) completing our analysis with the partial rate correlation function (PRCF, Berryman \& Turchin 2001), analogous to a 2-dimensional embedding in the RA framework.

The PRCF has been elaborated to detect densitydependent structures (thus high-frequency processes), by investigating the ( $d$-lagged) correlations between the rate of change $R_{t}=N_{t}-N_{t-1}$ and $R_{t-d}$, with the effect of earlier lags removed. Computing the autocorrelation of the rate of change $\left(R_{t}\right)$ rather than of the abundance $\left(N_{t}\right)$, as with the partial rate autocorrelation function (PACF), avoids any masking effects caused by positive autocorrelation due to reproduction (Berryman \& Turchin 2001). Its significance can be assessed directly without bootstrapping, using Bartlett's criterion, $\pm 2 n^{-0.5}$, with $n$ the length of the series (Box \& Jenkins 1976, Royama 1992). As seen above, the null model of the PRCF is a population subject to unbounded growth (i.e. a reddened noise), which should be preferred to the PACF null model (i.e. white noise). This would be further consistent with the bluefin historical catches, which display long-term (reddened) fluctuations (Ravier \& Fromentin 2001). This, in addition to its ability to uncover short-scale patterns (typically over a few lags), influenced us to include the PRCF as a complimentary step in the RA analysis of these series.

Data. Simulated examples - three elementary stochastic models: For illustration and testing purposes of the RA, we firstly simulated time series from 3 elementary stochastic and non-linear models that are currently used or referred to in ecology. The first model is a simple autoregressive process generating a random walk (i.e. a brown noise):

$$
N_{t+1}=N_{t}+\varepsilon_{t}
$$

where $\varepsilon_{t}$ is a gaussian white noise. This model simply corresponds to a population subject to unbounded growth (Fig. 1a) and therefore defines the null model in the sense of Berryman \& Turchin (2001). The second time series was derived from a stochastic Order 2 autoregressive model:

$$
N_{t+1}=\exp \left(a N_{t}+b N_{t-1}+\varepsilon_{t}\right)
$$

This model depicts how a delayed autoregressive process can be affected by an observation process of the exponential family (Fig. 1b). Non-linearity is attributed here to the exponential function which amplifies the random fluctuations of the autoregressive process, and would require log-transforming the series based on some a priori assumptions (Fromentin et al. 2001). Lastly, a third non-linear time series was constructed by adding gaussian noise to a simple 2-species trophic model, classically called NPZ model in plankton ecology (e.g. Franks et al. 1986):

$$
\begin{gathered}
N=N_{\text {tot }}-P-Z \\
\frac{\mathrm{d} P}{\mathrm{~d} t}=\frac{u P N}{N+N_{0}}-g \frac{Z P}{P+P_{0}} \\
\frac{\mathrm{d} Z}{\mathrm{~d} t}=a g \frac{Z P}{P+P_{0}}-\mathrm{k} Z
\end{gathered}
$$

where $N$ is the nutrient concentration, and $P$ and $Z$ the phytoplankton and zooplankton abundance, respectively. Parameters are set as follows: $N_{\text {tot }}=5$ (initial nutrient concentration), $N_{0}=0.1$ (nutrient threshold), $P_{0}=0$ (initial phytoplankton abundance), $u=0.3$ (uptake factor or growth rate of $P$ ), $g=0.2$ (grazing factor), $a=0.4$ (grazing conversion factor), $k=0.08$ (death rate of $Z$ ). The model is supposed to be partially observable, i.e. only the phytoplankton variable $P$ is measured, and blurred with white observation noise $W \sim N(0,1)$. This parameterization generates an oscillatory behaviour in the system and a series of cycles in the measured $(P)$ time series (Fig. 1c).

The embedding procedure implies that the full complexity of the system may be retrieved from the data sets. In other words, time series must be long enough for the results to be robust. We therefore tested RA with various lengths of each simulated time series, from 50 points up to 500 points.

Historical time series of bluefin tuna catches: Time series of bluefin tuna catches (in number of fish) have recently been collected after intensive work on 

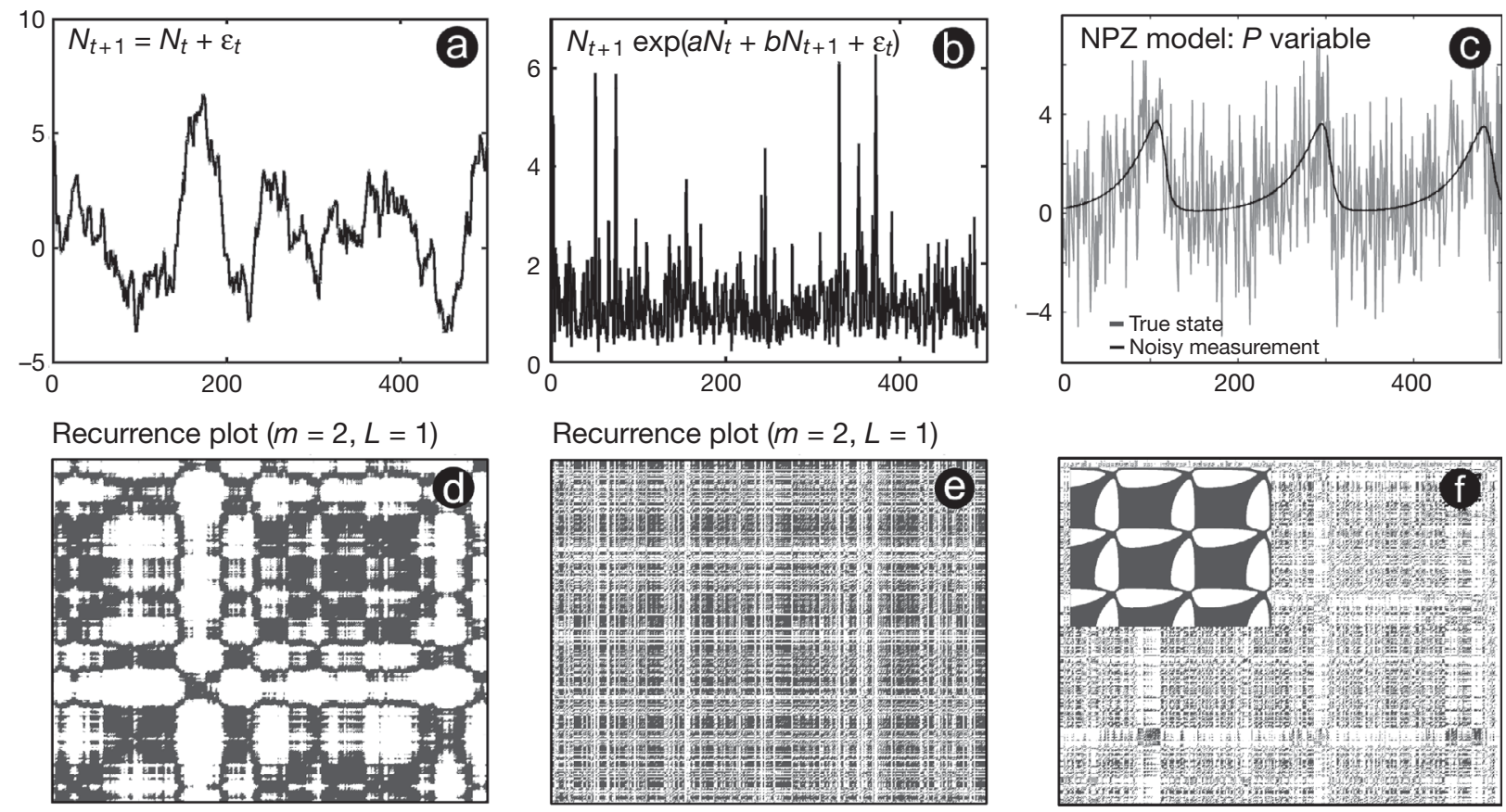

Fig. 1. Time series of 3 elementary stochastic models: (a) random walk, (b) exponentially transformed autoregressive process, and (c) noisy NPZ model, together with their corresponding recurrence plots $(\mathrm{d}-\mathrm{f}$ ) computed with an embedding dimension of 2 and time delay 1. (f) Recurrence plot of non-noisy (i.e. fully deterministic) NPZ model is inserted into recurrence plot of noisy NPZ model

archives and historical material (Ravier \& Fromentin 2001). These were derived from the Mediterranean and Atlantic costal trap fishery, which used a fixed gear to intercept bluefin tuna during their spawning migration (Fromentin \& Powers 2005). This original dataset spans a 360 yr period, from 1600 to 1960 , with notable gaps between 1820 and 1850. The configuration and mode of operation of these traps remained quite stable during that period (Farrugio 1981), and they all displayed synchronous variations at dominant periods of $\sim 20$ and $\sim 100$ to $120 \mathrm{yr}$, the latter accounting for 50 to $70 \%$ of the total variance. This indicated that a common process was likely to be observed, and that trap catches were likely to reveal long-term fluctuations in the abundance of migrating bluefin tuna (Ravier \& Fromentin 2001, 2004).

Although we computed a synthetic, full-length time series in previous studies (Ravier \& Fromentin 2001, 2004), we chose to apply RA to the original (shorter) series here, as recurring patterns were likely to have been smoothed or lost during this process. Following tests on the minimum needed length and results of Ravier \& Fromentin (2004), we selected time series displaying at least 125 contiguous years without missing values (Fig. 2). The 6 retained series came from Sicilian traps (Formica, Favignana and Bonagia: 180 annual values of catches from 1634 to 1813) and from Sardinian traps (Porto-Paglia, Porto-Scuso and Isola-Piana: 136 values from 1825 to 1960). The unusual length of these time series also allowed us to assume that the bluefin tuna population varied within a large range of abundance levels and patterns (i.e. states), which is a prerequisite for phase-space reconstruction and nonlinear analysis.

\section{RESULTS}

\section{Simulated examples: analysis of elementary stochastic models}

For comparison purposes, the 3 test time series were embedded in phase space of Dimension 2 and unit time delay. The corresponding RPs (Fig. 1) are easily distinguishable, displaying a number of vertical, horizontal and diagonal features. The random walk model is characterized by checker-board like patterns (Fig. 1d), while the exponential autoregressive process tends to fill up the RP with neighbouring points (Fig. 1e). While the deterministic fluctuations of the $P$ compartment of the last model are clearly visible in the form of sharp structures, once blurred by observation noise, they become more similar to the squared patterns of the 2 previous models (Fig. 1f). Still, the succession of the 3 cycles remains visible, separated by horizontal and vertical white strips indicating abrupt variations in the state vector.

We then tested these 3 elementary time series against the different types of surrogates using a hierar- 

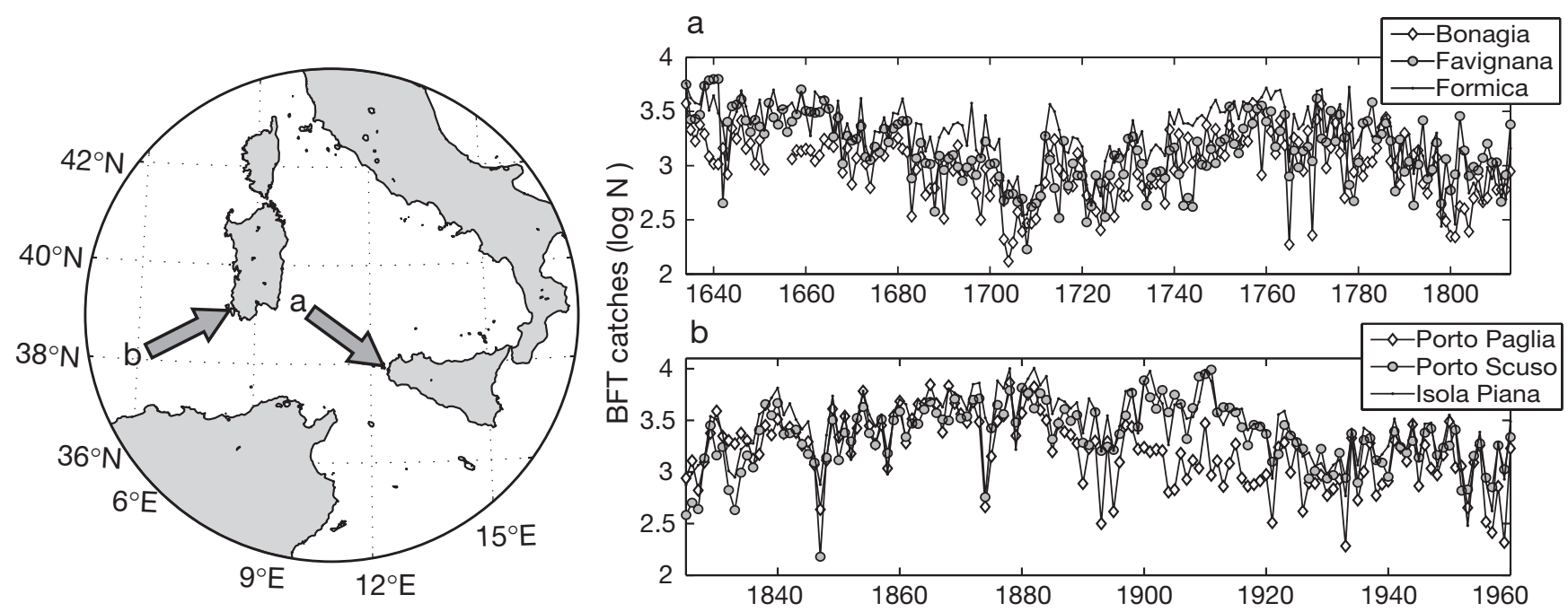

Fig. 2. Historical time series of bluefin tuna (BFT) fluctuations derived from (a) Sicilian and (b) Sardinian traps (after Ravier \& Fromentin 2001)

chical approach; i.e. we firstly tested against Type 0 surrogates: if $H_{0}$ was rejected we then tested against Type 1, and if it was rejected again we tested against Type 2 surrogates (all the results being summarised in Table 2). Note that a relatively low number of surrogates (20 to 30 ) proved sufficient for obtaining reliable results.

The test of the random walk model, which mostly displays long-term fluctuations, against Type 0 surrogates (shuffling the data in time domain) led to rejection of the null model in all cases. When these surrogates were then generated by randomly shuffling the phases of the Fourier transformed data (Type 1 surrogates), the RP-based statistics were all indistinguishable from their surrogate counterparts, except for the laminarity index (using the non-parametric test introduced earlier, $2 \alpha^{-1}-1$, with $\alpha=0.05$; see Table 2 ). Type 1 surrogates allow us to correctly identify the stochastic nature of the random walk and, thus, to avoid the attribution of these pseudo-cycles to deterministic processes or external forcing (which may have been tempting at first sight).

Table 2. Significance $\left({ }^{*}\right.$, at $95 \%$ ) of recurrence statistics for 3 elementary models. Each test was performed using embedding dimension $m=2$ and lag $L=1$. RW: random walk; T: Type

\begin{tabular}{|lllllllllll|}
\hline & \multicolumn{1}{c}{ Model 1 (RW) } & \multicolumn{1}{c}{ Model 2 (expRW) } & \multicolumn{3}{c|}{ Model 3 (NPZ) } \\
& T0 & T1 & T2 & T0 & T1 & T2 & T0 & T1 & T2 \\
\hline \%REC & $*$ & - & - & $*$ & $*$ & - & $*$ & $*$ & $*$ \\
$\%$ DET & $*$ & - & - & $*$ & $*$ & - & $*$ & $*$ & $*$ \\
$\%$ ADL & $*$ & - & - & $*$ & $*$ & - & $*$ & $*$ & - \\
LDL & $*$ & - & - & $*$ & $*$ & - & $*$ & $*$ & $*$ \\
LAM & $*$ & $*$ & - & $*$ & $*$ & - & $*$ & - & - \\
TT & $*$ & - & - & $*$ & $*$ & - & $*$ & - & - \\
\hline
\end{tabular}

The exponential of an autoregressive process, that appeared close to white noise but exhibited further occasional bursts (Fig. 1b,e), led to rejection of null model based on Type 0 or Type 1 surrogates with high confidence $(p<0.05$ for \%REC, \%DET, LDL, ADL, LAM and TT). This indicated that such a time series could not be assimilated to a 'white' or a 'reddened' noise. However, the test against Type 2 surrogates was not rejected, as no statistic could be differentiaed from their surrogate counterparts. Therefore, the exponential of an autoregressive process was consistent with red noise (unregulated growth) combined with a nonlinear observation function (i.e. a static non-linearity).

The NPZ model is a mix of deterministic and stochastic processes (Fig. 1c). Type 0 surrogates (time shuffling) retain the time distribution but destroy both the frequency distribution and any dynamic structure (Fig. 3a). The RP of such surrogates also appear to be quite different from that of the NPZ model (Figs. 1f \& 3d) and, as expected, this null model was rejected for all statistics (Table 2). Type 1 surrogates (phases shuffling) retain the frequency distribution (Fig. 3b) and display pseudo-cycles similar to those observed in the NPZ model. However, such variations do not appear to be as regular as in the original data, and the RPs also appear to be different (Figs. 1f \& 3e). Type 2 surrogates retain the frequency distribution and the time distribution (Fig. 3c). Nonetheless, its corresponding RP also appeared to be different from the original data, and both null models (Type 1 and Type 2) were rejected for the NPZ model for some statistics (Fig. 3f). Indeed, a significantly greater number of recurring patterns (\%REC), with a higher prediction time (\%LDL), were observed in comparison to red noise surrogates (Table 2). This indicated that the succession of the 3 


\section{NPZ model}

Type 0 surrogate
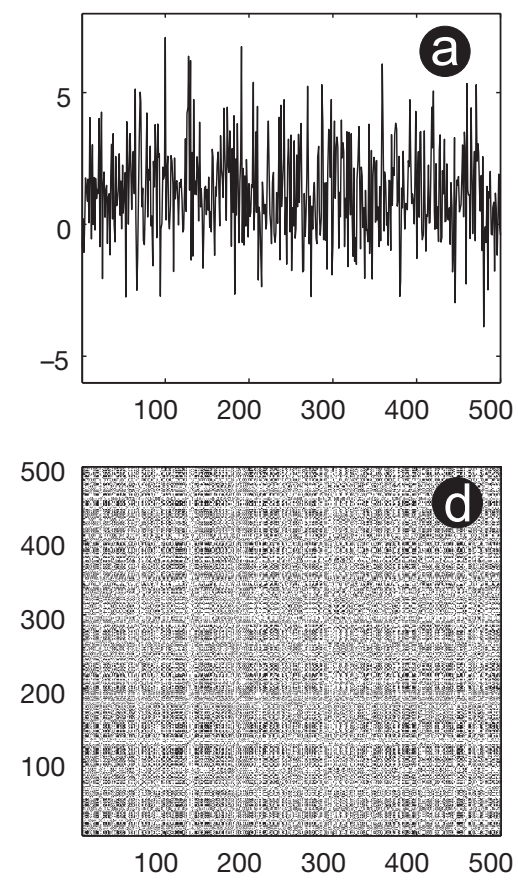

Type 1 surrogate
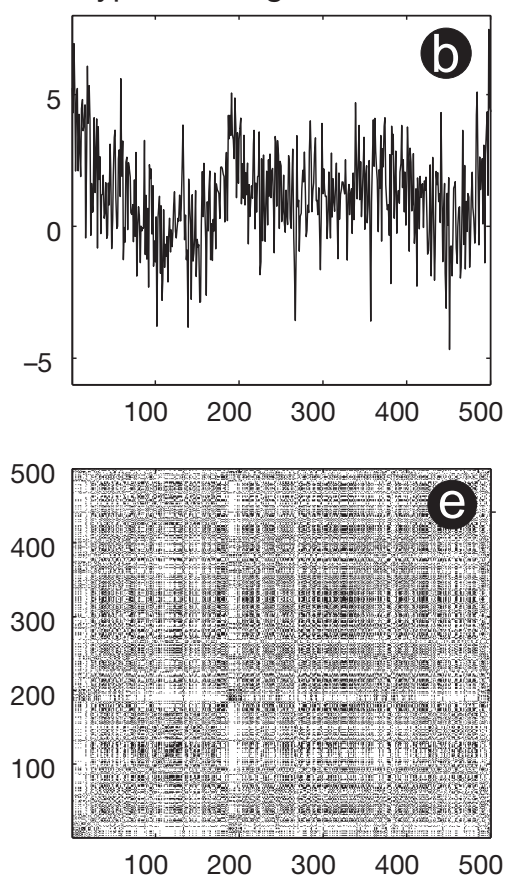

Type 2 surrogate
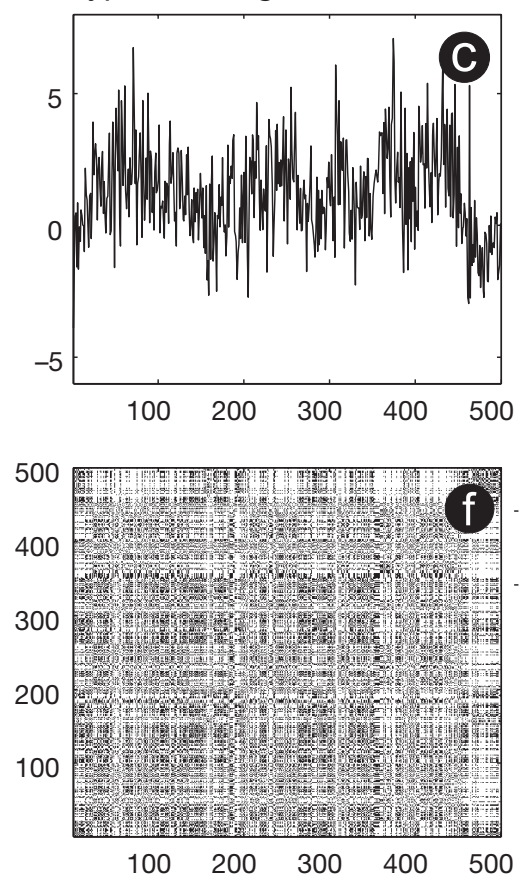

Fig. 3. (a) Type 0 (time-shuffled), (b) Type 1 (phase-shuffled), and (c) Type 2 (constrained phase-randomised Fourier transform) surrogates corresponding to noisy NPZ model. Corresponding recurrence plots $(\mathrm{d}-\mathrm{f})$ were computed with an embedding dimension of 2 and time delay 1

cycles (visible on the RP, Fig. 1f) did not result from stochastic processes but from deterministic ones.

\section{Recurrence analysis of bluefin tuna time series}

Given the global synchrony between the Mediterranean traps (Ravier \& Fromentin 2001), embedding parameters were set to be identical for the 6 time series. False nearest neighbours advocated an embedding dimension $m$ of about 8 , while the first minimum in the self-mutual information function favoured small time-delays (i.e. $L \sim 1$ to 3 ). As fishing activity occurred every year during spawning migration (i.e. May to July), it can be hypothesized that the frequencies of the observation and the ecological processes were closely matching; therefore, we used a time delay $L$ of 1 . This avoided sub-sampling the data (the full time series were used), but introduced the risk is of including observation noise in the embedding process. Not surprisingly, the null hypotheses of white noise (i.e. Type 0 surrogates) was rejected for all time series and for all recurrence statistics.

Less trivially, the same results were obtained with reddened noise (i.e. Type 1 surrogates). In other words, the catch time series showed significantly more recur- ring patterns than implied by a white null model (uncorrelated random fluctuations) or a reddened null model (autoregressive model). To illustrate this result, examples of surrogates are given for the Formica time series in Fig. 4. Type 0 and Type 1 surrogates indeed appear to be quite different to the original structure of the observed time series, but Type 2 surrogates look more similar. However, the null model that preserves both the original non-gaussian distribution of the trap time series and their reddened power spectra (Type 2 surrogates) only allowed a partial number of recurring features of the various trap time series to be uncovered (Table 3). All the time series displayed significant differences $(p<0.05)$ to the null model for given statistics (bearing in mind that these were different among the traps). For example, Formica, Favignana, Bonagia and Piana displayed significantly more recurring vectors than the null model (Table 3). Favignana and Piana also displayed more deterministic structures, and Favignana and Bonagia larger laminar time periods, than what would be expected under the null model. Note also that Bonagia exhibited a greater average diagonal length, which could indicate a longer mean prediction time. Porto-Scuso displayed a significantly larger prediction horizon (LDL), perhaps indicating dynamics of lower complexity. Although the 6 trap time series 
Recurrence plot Formica $(m=8, L=1)$
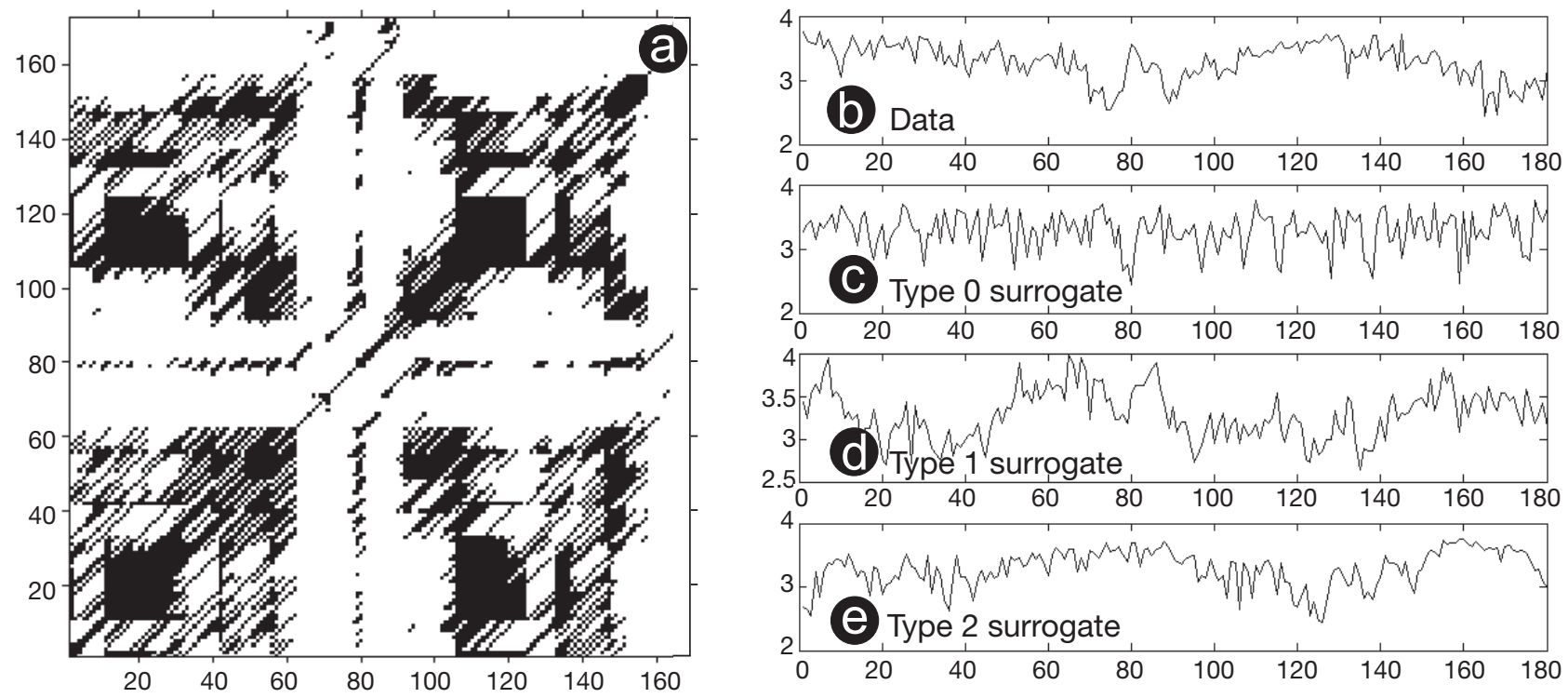

Fig. 4. (a) Recurrence plot of Formica time series with an embedding dimension of 8 and time delay 1. Examples of surrogates are given on right: (b) original log-transformed data time series, (c) corresponding Type 0 surrogate (time-shuffled), (d) corresponding Type 1 surrogate (phase-shuffled), and (e) corresponding Type 2 surrogate (constrained phase-randomised Fourier transform)

exhibited different results, Favignana and Bonagia displayed quite similar statistics, being different to the third Sicilian trap (Formica) and the 3 Sardinian ones. Among the Sardinian traps, Porto-Paglia and PortoScuso appeared to be most similar, while Isola Piana displayed some recurrent patterns close to those of the Sicilian traps.

\section{Partial rate autocorrelation function}

To complete the RA, the partial rate autocorrelation was computed for the first-order finite difference of all 6 series. The PRCF plots displayed negatively correlated autocorrelation in $R_{t}$ over 2 successive time lags (i.e. of Order 2). This result was true for the 6 catch

Table 3. Significance $\left({ }^{*}\right.$, at $\left.95 \%\right)$ of recurrence statistics for 6 bluefin tuna catch time series from coastal Mediterranean traps. Each test was performed against surrogates of Type 2 (i.e. stochastic autoregressive process with static nonlinearity) using embedding dimension $m=8$ and $\operatorname{lag} L=1$. Row definitions as in Table 1

Statistic Formica Favignana Bonagia Paglia Scuso Piana

\begin{tabular}{lllllll}
\hline \%REC & $0.04^{*}$ & $0.00^{*}$ & $0.00^{*}$ & 0.16 & 0.14 & $0.02^{*}$ \\
$\%$ DET & 0.11 & $0.04^{*}$ & 0.16 & 0.47 & 0.19 & $0.04^{*}$ \\
ADL & 0.47 & 0.11 & $0.04^{*}$ & 0.28 & 0.35 & 0.28 \\
LDL & 0.39 & 0.11 & 0.18 & 0.12 & $0.02^{*}$ & 0.76 \\
LAM & 0.72 & $0.00^{*}$ & $0.00^{*}$ & 0.11 & 0.25 & 0.28 \\
TT & 0.61 & 0.06 & 0.14 & 0.11 & 0.09 & 0.67 \\
\hline
\end{tabular}

time series considered here (Fig. 5), as well as for the other smaller series in the original data set (results not shown). The fact that significant negative autocorrelation is a common feature of all trap catch time series would, thus, strongly advocate for density-dependent structures in migrating bluefin tuna. The occurrence of density-dependence could explain why we previously found more recurring/deterministic structures than the Type 2 null model in several time series (see above or Table 2). Weakly significant correlations could be detected at other lags according to Bartlett's criterion (e.g. Lag 11 for Formica, 10 for Bonagia, 4 for Porto Paglia, 3 for Porto Scuso, and 3, 7 and 10 for Isola Piana). However, only the negative direct and delayed correlations (Lags 1 and 2) appeared significant for all time series.

\section{DISCUSSION}

\section{Usefulness and significance of recurrence analysis in ecology}

The recurrence statistics proposed by Zbilut (2002) and Marwan et al. (2002) can be seen as new 'probes' of complexity (sensu Turchin 2003) for ecological time series. Recurrences can be used on raw data analysis and are complementary to standard time series tools such as autocorrelation functions or the Fourier spectrum - that provide clues about the ecological 'smoothness' of underlying processes (Chatfield 1999). 

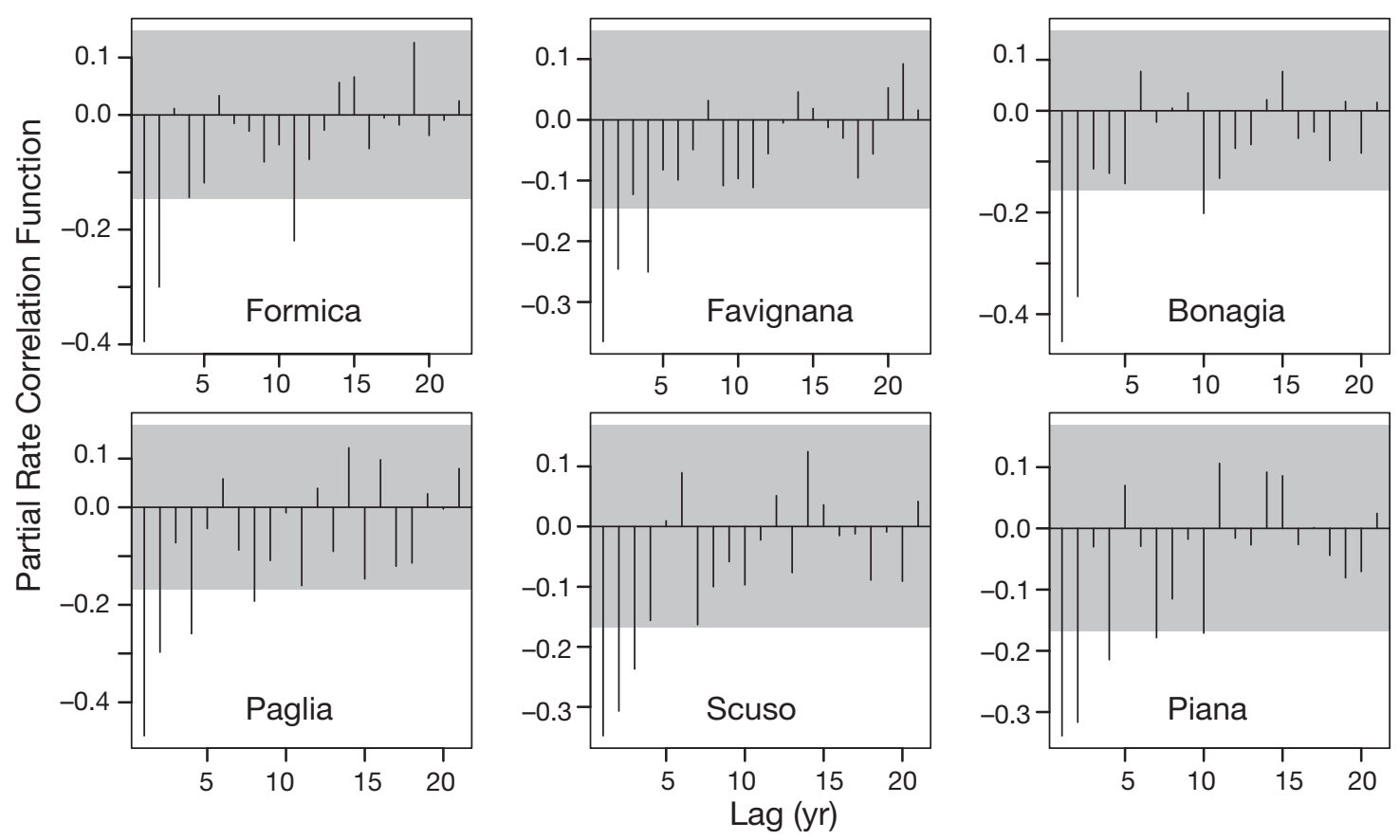

Fig. 5. Partial rate correlation functions (PRCFs) for 3 Sicilian (Formica, Favignana, Bonagia) and 3 Sardinian (Porto-Paglia, PortoScuso and Isola-Piana) time series. Confidence band (grey area) given by Bartlett's criterion $\left( \pm 2 n^{-0.5}\right)$

According to the algorithmic definition of randomness (Chaitin 2001), recurring patterns are highly informative regarding the degree of stochasticity in a data set. However, the qualitative information produced by the $\mathrm{RP}$, although useful, remains insufficient (and somewhat too subjective) to allow interpretation of time series. Therefore, recurrent analyses must also include the computation of RP-based statistics. Furthermore, we think that these statistics have to be tested against clear null models and thus proposed 3 types of surrogates. The use of surrogates indeed provided a convenient answer to the question: 'how much deterministic structure is embedded in a time series?'. In that sense, surrogate-based RA is nothing more than hypothesis testing, i.e. we assume a 'trivial' option to be true unless we can reject it by finding significant structure in the data. Using 3 elementary and simulated time series, we showed that this procedure was quite powerful and able to distinguish between pure stochastic process (i.e. the random walk) and the mixture of stochastic and deterministic ones (i.e. the noisy autoregressive model and the NPZ model). Doing so, we can then identify the models most likely relevant to an ecological time series. For instance, a state-space formulation may be relevant for the noisy autoregressive example, which may not be obvious at first glance (i.e. bursts could be interpreted as dynamic non-linearity). Finally, we are limited by the degree of complexity of the null hypotheses that we can propose. To date, the simplest (or most trivial) processes we are able to generate by constrained simulation belong to the linear or weakly non-linear class (e.g. surrogates of Type $0,1,2)$. Recent efforts on constrained randomization broaden the repertoire of such null hypotheses. Generally, random processes blur deterministic dynamics through observation noise (generally additive) and dynamical noise (possibly propagated through the system).

Quantifying the resulting variability through RA can help in separating both sources, acknowledging that no technique is noise-insensitive. RA is subject to such noise through the choice of $r$; for our specific application to bluefin tuna, we used the partial rate autocorrelation function to complete the analysis. Knowing that ecological time series are often of limited length (especially with in situ data), we tested the sensitiveness of the RA analysis to time span. Using simulated data, we repeated the RA steps after truncating a time series of 500 values in sub-samples of length 50,100, 150, 200, 300 and 400. Boxplots of the results (1 plot for each RPbased statistic) were similar until a length of $\sim 100$ to 150 values (after which some structures were lost). However, such a limit remains only indicative and depends on the ratio of deterministic structures versus noise. Most RP statistics appeared to be invariant over time or displayed a plateau-like behavior, except for statistics associated with the length of some event (e.g. LDL and LAM), which grew almost linearly with the series length, especially for the random walk model (results not shown). 


\section{Historical fluctuations in bluefin tuna catches}

RA was applied to the historical time series of bluefin tuna catches from the Mediterranean coastal traps. A dimension of 8 was found for phase space embedding, while testing against surrogates indicated more recurring features than expected from a linear stochastic process. While the lengths of these series were at the lower limit of what is needed for reliable results, the results among the 6 chosen series were globally homogeneous and significant. Thus, this analysis confirms (through a quantitiative and objective test) the nonstochastic nature of long-term fluctuations in bluefin tuna trap catches, as postulated by Ravier \& Fromentin (2001, 2004). In particular, hypothesis testing using Fourier-based surrogates revealed that the observed dynamics could not be described solely by their frequency spectrum and time distribution: most time series displayed more recurring patterns than expected under this null hypothesis.

These results are in agreement with the general view that marine populations display non-linear stochastic variations (Sugihara 1994, Hsieh et al. 2005). In particular, Hsieh et al. (2005) showed that time series of commercial landings of Pacific salmon, trout and zooplankton (the latter obtained from CalCOFI surveys) were characterized by embedding dimensions of 3 to 8 , an indication of low dimensional non-linear processes. On the other hand, physical variables such as sea surface temperature and climatic oscillation indices were characterized by high and very high dimensions (10 to 20), indicating that high-order linear stochastic processes are better at capturing their variations. A dimension of 8 for the bluefin tuna time series is coherent with the range computed by these authors for their biological series, and would advocate for low dimensional non-linear processes. This view is in partial agreement with the hypothesis of 'cohort resonance' as a possible mechanisms to generate low frequency oscillations when many age classes interact (Fromentin 2002, Bjornstad et al. 2004).

Following this, the bluefin tuna population would act as low-pass filter (a smoother) of variability affecting critical processes, such as recruitment or growth at early stages. However, such autoregressive process still posses a linear basis and produce random longterm variations rather than deterministic (recurrent) patterns. This hypothesis can be extended in light of our results: it is suggested that a blend of environmental forcing and non-linear biological responses occurs at time scales of 10 to $30 \mathrm{yr}$, which is in the order of the life span of Atlantic bluefin tuna (Mather et al. 1995). Atmospheric forcing (synthesized through e.g. the North Atlantic Oscillation) could therefore affect bluefin tuna population through the occurrence and strength of meso-scale processes where spawning is known to occur (Garcia et al. 2003). As bluefin tuna displays a 'homing-like' behavior and quickly switches from a wide geographical distribution to restricted spawning areas (Fromentin \& Powers 2005), such atmospheric forcing is likely to induce non-linear responses. These delayed interactions may remain hidden to classic correlation-based analysis and would therefore need to be investigated through mechanistic age-structured models. Possible interactions with local units in a metapopulations context could also lead to non-linearity in the response to climate forcing (Fromentin \& Powers 2005).

As RA may yield less reliable results at very small scales (due to e.g. high observation noise), we completed the methodology with the computation of PAFC (a simplified form of phase-space embedding). A significant negative feedback was uncovered for all 6 time series, at Lags 1 and 2. Such feedback is usually defined in ecological theory as a density-dependence, and is seen as a necessary condition for population regulation in general (Berryman 2002). Density-dependence theory supposes a negative relationship between population abundance $\left(N_{t}\right)$ and its growth rate $\left(R_{t}\right.$ a function of per-capita birth, death, immigration and emigration rate). As Atlantic bluefin tuna spawn in restricted areas in the Mediterranean (Fromentin \& Powers 2005), one could first see resource/space limitations as a possible explanation (bluefin larvae reach an active and voracious swimming stage after only $2 \mathrm{wk}$, see Mather et al. 1995) or even through cannibalism (within early stages or through predation by adults on age-0 classes). High densities of Atlantic bluefin tuna larvae or cannibalism have not been reported, but such observation is difficult to collect and remains scarce (Garcia et al. 2003, Fromentin \& Powers 2005). Note, however, that high density of tuna larvae has been already observed for a neighbouring species, the southern bluefin tuna Thunnus maccoyii (T. Smith pers. comm.). Densitydependent processes appear to be less probable within the adult stages because of their wide spatial distribution and highly migratory behavior, so that local depletion in forage or changes in predation (e.g. by killer whales Orcinus orcas) are more likely to induce changes in migration patterns than in abundance (e.g. Tiews 1978). Also. the naturally high unpredictability of small pelagic populations (Spencer \& Collie 1997) does not lend much support to such deterministic hypothesis. An alternative to densitydependence in early stages would involve non-yearly spawning (a feature that has been observed in bluefin tuna in captivity, see Lioka et al. 2000).

According to the observed Order 2 negative feedback, a significant portion of migrants coming from the 
Atlantic would therefore only spawn in the Mediterranean every $3 \mathrm{yr}$, which can be related either to: (1) a physiological inability of adults to perform gonad maturation every year, or (2) the frequentation of other spawning grounds (as already postulated by various authors, e.g. Lutcavage et al. 1999, Ravier \& Fromentin 2004). This hypothesis can be further interpreted in the framework of marine metapopulations, a concept shown to be highly relevant in the case of bluefin tuna (Fromentin \& Powers 2005). Synchrony within the fraction of migrants may then be explained through stabilizing mechanisms similar to the 'school-trap hypothesis' (Bakun \& Cury 1999). However, there is little knowledge on the maturation costs of gonads in spawning bluefin tuna. While there is evidence of rapid maturation in relation to warmer temperatures once tuna are in the Mediterranean (Medina et al. 2002), year-to-year changes have not been reported and may lack physiological basis (M. Lutcavage pers. comm.). Nonetheless, such delayed negative feed-back remains highly significant, and hypotheses need now to be built and tested using alternative sources of data.

\section{CONCLUSION}

Disentangling the effects of endogenous and exogenous processes in ecology is an important field of research. In this study, we presented a method that can uncover deterministic features in ecological time series. Recurrent features form part of the most basic clues about the structure of a biological system (e.g. reproduction patterns). Using phase-space embedding and recurrences as a signature for determinism, we could test whether observed fluctuations were consistent with linear stochastic surrogates. Such analysis can provide clues on the underlying complexity of measured signals (e.g. whether a linear or non-linear model should be used, whether internal stochastic processes suffice to induce the observed variability...). The application of this method to historical time series of bluefin tuna catches revealed that long-term fluctuations could not be assimilated to simple stochastic or autoregressive processes. Small-scale variability was addressed using PAFC, and we further uncovered negative feedback of Orders 1 and 2 in all time series, indicating either density-dependence processes during early stages, or non-yearly spawning. We provided suggestions for further research into the modelling of these patterns. In particular, the mechanistic modelling of responses of bluefin tuna to oceanic variability should be considered, using age structured and timedelay formulations. On the other hand, the origin of density-dependent patterns should be investigated using a hypothesis testing approach.
Acknowledgements. We thank 4 anonymous referees who helped to improve the clarity of this manuscript. Bernard Cazelles also provided valuable and helpful comments on an earlier version. This study was conducted as part of a $\mathrm{PhD}$ project by F.R., co-funded by CLS (Collecte Localisation Satellites, Ramonville-St-Agne, France) and IFREMER (Institut Francais de Recherche pour l'Exploitation de la Mer).

\section{LITERATURE CITED}

Bakun A, Cury P (1999) The 'school trap': a mechanism promoting large-amplitude out-of-phase population oscillations of small pelagic fish species. Ecol Lett 2:349-351

Berryman A (2002) Population regulation, emergent properties, and a requiem for density dependence. Oikos 99: $600-606$

Berryman A, Turchin P (2001) Identifying the density-dependent structure underlying ecological time series. Oikos 92: 265-270

Bjornstad O, Nisbet R, Fromentin JM (2004) Trends and cohort resonant effects in age-structured populations. J Anim Ecol 73:1157-1167

Boudjema G, Chau NP (1996) Revealing dynamics of ecological systems from natural recordings. Ecol Model 91:15-23

Boudjema G, Cazelles B (2001) Extraction of nonlinear dynamics from short and noisy time series. Chaos Soliton Fract 12:2051-2069

Box GEP, Jenkins GM (1976) Time series analysis, forecasting and control. Holden Day, San Francisco, CA

Cazelles B, Boudjema G (2001) The Moran effect and phase synchronization in complex spatial community dynamics. Am Nat 157:670-676

Chaitin GJ (2001) Exploring randomness. Springer-Verlag, London

Chatfield C (1996) The analysis of time series-an introduction, 5th edn. Chapman \& Hall, London

Cuddington KM, Yodzis P (1999) Black noise and population persistence. Proc R Soc Lond B 266:969-973

Dale MRT, Dixon P, Fortin MJ, Legendre P, Myers DE, Rosenberg MS (2002) Conceptual and mathematical relationships among methods for spatial analysis. Ecography 25: 558-577

Dippner JW, Heerkloss R, Zbilut JP (2002) Recurrence quantification analysis as a tool for characterisation of nonlinear mesocosm dynamics. Mar Ecol Prog Ser 242:29-37

Eckmann JP, Kamphorst SO, Ruelle D (1987) Recurrence plots of dynamical systems. Europhys Lett 5:973-977

Farrugio H (1981) Exploitation et dynamique des populations de thon rouge, Thunnus thynnus (Linné 1758) AtlantoMéditerranéennes. PhD dissertation, Université des Sciences et Techniques du Languedoc, Montpellier

Franks PJS, Wroblewski JS, Flierl GR (1986) Behavior of a simple plankton model with food-level acclimation by herbivores. Mar Biol 91:121-129

Fromentin JM (2002) Can stochastic variations in recruitment induce long-term fluctuations in the carrying capacity? Collect Vol Sci Pap ICCAT 54:985-991

Fromentin JM, Powers JE (2005) Atlantic bluefin tuna: population dynamics, ecology, fisheries and management. Fish Fish 6:281-306

Fromentin JM, Myers RA, Bjornstad ON, Stenseth NC, Gjosaeter J, Hartvig C (2001) Effects of density-dependent and stochastic processes on the regulation of cod populations. Ecology 82:567-579

Gao J, Cai H (2000) On the structures and quantification of recurrence plots. Phys Lett A 270:75-87 
Garcia A, Alemany F, Velez-Belchí P, López Jurado JL, de la Serna JM, González Pola C, Rodriguez JM, Jansá J (2003) Bluefin tuna and associated species spawning grounds in the oceanographic scenario of the Balearic archipelago during June 2001. Collect Vol Sci Pap ICCAT 56:138-148

Gurney WS, Nisbet R (1998) Ecological dynamics. Oxford University Press, Oxford

Halley JM (1996) Ecology, evolution, and 1/f noise. Trends Ecol Evol 11:33-37

Hasselmann K (1976) Stochastic climate models I: theory. Tellus 28:473-485

Hilborn R, Mangel M (1997) The ecological detective: confronting models with data. Princeton University Press, Princeton, NJ

Hsieh C, Glaser SM, Lucas AJ, Sugihara G (2005) Distinguishing random environmental fluctuations from ecological catastrophes for the North Pacific Ocean. Nature 435:336-340

Kantz H, Schreiber T (1997) Nonlinear time series analysis. Cambridge University Press, Cambridge

Lioka C, Kani K, Nhhala H (2000) Present status and prospects of technical developmment of tuna sea-farming. Cah Options Méditerranéennes 47:275-285

Lutcavage M, Brill R, Skomal GB, Bradford CC, Howey PW (1999) Results of pop-up satellite tagging of spawning size class fish in the Gulf of Maine: do North Atlantic bluefin tuna spawn in the mid-Atlantic? Can J Fish Aquat Sci 56:173-177

Marwan N (2003) Encounters with neighbours-current developments of concepts based on recurrence plots and their applications. $\mathrm{PhD}$ thesis, University of Potsdam

Marwan N, Wessel N, Meyerfeldt U, Schirdewan A, Kurths J (2002) Recurrence plot based measures of complexity and its application to heart rate variability data. Phys Rev E 66:026702

Mather FJ, Mason JM, Jones AC (1995) Historical document: life history and fisheries of Atlantic bluefin tuna, report No. 370. NMFS Southeast Fisheries Science Centre, Miami, FL

Matlab (2003) Matlab release 13, the language of technical computing. MathWorks, Natick, MA

Medina A, Abascal FC, Megina C, Garcia A (2002) Stereological assessment of the reproductive status of female Atlantic northern bluefin tuna during migration to Mediterranean spawning grounds through the Strait of Gibraltar. J Fish Biol 60:203-217

Morales JM (1999) Viability in a pink environment: why 'white noise' models can be dangerous. Ecol Lett 2:228-232

Editorial responsibility: Howard I. Browman (Associate Editor-in-Chief), Storebø, Norway
Parrott L (2004) Analysis of simulated long-term ecosystem dynamics using visual recurrence analysis. Ecol Complex 1(2):111-125

Ravier C, Fromentin JM (2001) Long-term fluctuations in the Eastern Atlantic and Mediterranean bluefin tuna population. ICES J Mar Sci 58:1299-1317

Ravier C, Fromentin JM (2004) Are the long term fluctuations in Atlantic bluefin tuna population related to environmental changes? Fish Oceanogr 13:145-160

Ripa J, Lundberg P, Kaitala V (1998) A general theory of environmental noise in ecological food webs. Am Nat 151:256-263

Rohani P, Miramontes O, Keeling MJ (2004) The colour of noise in short ecological time series data. Math Med Biol 21:63-72

Royama T (1992) Analytical population dynamics. Chapman \& Hall, London

Rudnick DL, Davis RE (2003) Red noise and regime shifts. Deep-Sea Res 50:691-699

Schreiber T, Schmitz A (1996) Improved surrogate data for nonlinearity tests. Phys Rev Lett 77:635-638

Small M, Tse CK (2003) Determinism in financial time series. Stud Nonlinear Dyn E 7:1-31

Spencer PD, Collie JS (1997) Patterns of population variability in marine fish stocks. Fish Oceanogr 6:188-204

Steele JH (1985) A comparison of terrestrial and marine ecological systems. Nature 313:355-358

Sugihara G (1994) Non-linear forecasting for the classification of natural time-series. Phil Trans R Soc A 348:477-495

Takens F (1981) Detecting strange attractors in turbulence. In: Rand DA, Young LS (eds) Dynamical systems and turbulence, Vol 898. Springer-Verlag, New York, p $366-381$

Theiler J, Eubank S, Longtin A, Galdrikian B, Farmer JD (1992) Testing for non-linearity in time series: the method of surrogate data. Phys D 58:77-94

Tiews K (1978) On the disapperance of bluefin tuna in the North Sea and its ecological implications for herring and mackerel. Rapp p-v Réun Cons Int Explor Mer 172:301-309

Turchin P (2003) Complex population dynamics: a theoretical/ empirical synthesis. Princeton University Press, Princeton, NJ

Vasseur DA, Yodzis P (2004) The color of environmental noise. Ecology 85:1146-1152

Zbilut JP, Thomasson N, Webber CL Jr (2002) Recurrence quantification analysis as a tool for nonlinear exploration of nonstationary cardiac signals. Med Eng Phys 24:53-60

Submitted: May 20, 2005; Accepted: January 9, 2006

Proofs received from author(s): July 21, 2006 